Published in final edited form as:

Top Magn Reson Imaging. 2005 December ; 16(6): 397-398.

\title{
Magnetic Resonance Imaging of Dementia
}

\author{
Michael W. Weiner, MD
}

When magnetic resonance imaging (MRI) was first developed for medical imaging, most physicians and investigators focused on brain disorders which could not be easily be diagnosed with x-ray computed tomography (CT) scanning. Multiple sclerosis was an obvious example because $\mathrm{x}$-ray CT did not provide sufficient soft tissue contrast to identify lesions in the white matter, whereas T2-weighted MRI highlighted these areas. The ability of MRI to identify brain tumors and stroke rapidly became apparent, and like x-ray CT, MRI has been extensively used to characterize diseases of the brain and other organs, which have identifiable lesions.

Until recently, there has been comparatively little interest in using MRI for the study of neurodegenerative diseases, including Alzheimer disease, other causes of dementia such as frontotemporal dementia and Lewy body disease, Parkinson disease, amyotropic lateral sclerosis, etc. There are several reasons for this.

First, the lack of interest on the part of the MRI community has reflected the relative lack of interest on the part of the medical community at large.

Second, the lack of interest was due to the fact that no treatments were available for neurodegenerative disease, thus, fostering the attitude "if you can't treat it, why diagnose it?"

Third, for the most past, neurodegenerative diseases are not characterized by lesions, although various types of lesions, especially in white matter, often occur in these conditions.

Fourth, neurodegenerative diseases do produce brain atrophy, due to loss of neuronal cell bodies and axons. However, the wide range of normal prevents diagnosis of Alzheimer and other neurodegenerative diseases, until the disease is very advanced, and the atrophy becomes extreme.

During the last decade, interest in MRI of neurodegenerative diseases has been rapidly rising. Much of this increased interest is due to the increase in the incidence and prevalence of these diseases, caused by the increase in the numbers of elders in the populations of the United States, Europe, and Japan. In addition, the imaging techniques have become more refined, especially due to the development of computer-assisted image processing tools with quantification of the volumes of brain structures. Finally, improved knowledge concerning the pathophysiological mechanisms of neurodegenerative diseases, especially Alzheimer disease, has led to the development of putative treatments which are entering clinical trials. The pharmaceutical companies have recognized that imaging techniques especially MRI and positron emission tomography provide "surrogate" information concerning the pattern and rate of neurodegeneration, which can be used to monitor the effects of treatments which slow the progression of neurodegeneration. All of these factors have led to an explosion of interest in imaging of neurodegenerative diseases, culminating in the funding of the Alzheimer's Disease Neuroimaging Initiative which is a very large multisite study using MRI, positron emission

\footnotetext{
${ }^{1}$ Reprints: Michael W. Weiner, MD, Center for Imaging of Neurodegenerative Disease, VA Medical Center (114M), University of California, San Francisco, CA (e-mail: michael.weiner@ucsf.edu)..
} 
tomography, and biomarkers, together with clincian measures, to monitor disease progression (further information at http://www.ADNI-info.org).

In this special issue of Topics in Magnetic Resonance Imaging, we present invited review papers dealing with several important topics in this area. The paper Magnetic Resonance Approaches to Brain Aging-Associated Neuropathology by Anderson and Kaye provides an overview of the topic. Dr DeCarli reviews an important topic in his paper Measurement and Impact of Concurrent Cerebrovascular Brain Injury in Alzheimer Disease. Drs Whitehall and Jack also deal with the issue of differential diagnosis in their paper Comparisons Between Alzheimer Disease, Frontotemporal Lobar Degeneration, and Normal Aging With Brain Mapping," and finally, Dr Wahlund presents An Evidence-based Evaluation of Magnetic Resonance Imaging as a Diagnostic Tool.

Finally, this observer of the scene predicts that the importance of this entire field will increase several orders of magnitude as soon as a treatment which slows the progression of Alzheimer disease is identified and announced. Several clinical treatment trials are coming to a close (results not yet announced), and many more are being planned; thus, we could read such an announcement of a successful treatment trial in our morning newspapers as early as next year or within the next few years. The implications then become obvious and huge. How do we assist the family physician in making the diagnosis of Alzheimer disease and distinguishing it from other dementias? What is the role of the neuroradiologist in diagnosing disorders not characterized by lesions? How do we use automated segmentation and registration programs for improved diagnosis? And finally, how do we identify those individuals who are currently cognitively healthy but who are at high risk for the future development of Alzheimer disease, and what is the role of MRI in early detection?

We, in this field, will have the opportunity to take advantage of all of the rapidly improving technologies of MRI acquisition (structural, perfusion, diffusion tensor, susceptibilityweighted MRI and magnetic resonance spectroscopic imaging), reconstruction (Fourier and Bayesian methods), and image processing (segmentation and registration) techniques to address these fascinating and very high-impact medical questions. 